\title{
PAEDIATRICS
}

\section{Autophagy in bladder exstrophy}

Patients with classic bladder exstrophy (CBE) in whom closure is delayed exhibit increased rates of bladder smooth muscle cell autophagy and apoptosis, and reduced rates of cellular proliferation, according to findings from a new study.

The pathophysiology and cellular basis of $\mathrm{CBE}$ is not well understood. In previous work, the researchers identified clear differences in bladder smooth muscle cell function between patients with CBE and controls. In their new study, Shabaninia et al. compared autophagy, apoptosis, and cell proliferation in patients undergoing early closure of exstrophy and those undergoing delayed closure.

The researchers obtained primary cultures of bladder smooth muscle cells from five patients undergoing primary early neonatal bladder closure (median age 5 days), five patients undergoing delayed bladder closure owing to a small bladder template at the time of birth (median age 1 year), and five control patients with vesicoureteral reflux who were undergoing open ureteral reimplantation (median age 4.25 years).

The team found that expression of the autophagy marker LC3 was significantly higher in the delayed closure group than in the other groups and found no differences in expression of LC3 between the neonatal early closure and control groups. Apoptotic indices of smooth muscle cells were also higher in the delayed closure group than in the other two groups. Expression of the cellular proliferation marker Ki67 was significantly lower in the delayed closure group than in the other two groups.

The authors state that their results emphasize the importance of early bladder closure in patients with CBE and say that manipulation of autophagy might become useful for improving outcomes in these patients.

Rebecca Kelsey

ORIGINAL ARTICLE Shabaninia, M. et al. Autophagy, apoptosis and cell proliferation in exstrophy-epispadias complex. Urology http://dx.doi.org/10.1016/j.urology.2017.09.015 (2017) 